\title{
Telemedicine for neuromuscular disorders during the COVID-19 outbreak
}

\author{
Matteo Garibaldi ${ }^{1}\left[\right.$ [ Gabriele Siciliano ${ }^{2} \cdot$ Giovanni Antonini $^{1}$
}

Received: 6 May 2020 / Revised: 2 July 2020 / Accepted: 6 July 2020 / Published online: 10 July 2020

(c) Springer-Verlag GmbH Germany, part of Springer Nature 2020

\section{Dear Sirs,}

Telemedicine (TM), whose potential use in disasters and public health emergencies has been highlighted in recent years [1], is now a worldwide rapidly emerging approach for outpatient care in pandemic era [2,3]. During the COVID19 outbreak, the volume of elective or non-urgent outpatient cares has significantly been reduced in many centers to limit infection exposure to patients, communities and medical staff, reserving face-to-face consultations to clinical relapses, emergencies or hospital therapies. In this context, TM has been widely proposed as one of the best alternatives for ambulatory clinical practice during quarantine [4].

Studies across the vast array of neurological disorders have reported the role of TM in providing access to neurologic care to people, otherwise limited by geographic or physical barriers [5]. During the COVID-19 lockdown, several neurological societies and independent study groups have developed documents for care delivery to patients affected by neurological and, in particular, neuromuscular diseases (NMD), highlighting the need of new clinical structures including robust telemedicine platforms and procedures to care for NMD patients [6]. Noteworthy, most NMD are chronic, slowly progressive and usually managed

Matteo Garibaldi

matteo.garibaldi@uniroma1.it

Gabriele Siciliano

gabriele.siciliano@unipi.it

Giovanni Antonini

giovanni.antonini@uniroma1.it

1 Department of Neuroscience, Mental Health and Sensory Organs (NESMOS), Faculty of Medicine and Psychology, Neuromuscular and Rare Disease Centre, Sant'Andrea Hospital, SAPIENZA University of Rome, Via di Grottarossa 1035-1039, 00189 Rome, Italy

2 Department of Clinical and Experimental Medicine, Neurological Clinic, University of Pisa, Via Savi 10, 56126 Pisa, Italy in outpatient care services, which makes these disorders particularly suitable for TM. However, remote clinical evaluation can have some important limitations. First of all, while TM assessment appears appropriate for NMD patients with known diagnosis and fairly stable clinical condition, it loses power when used for diagnostic purposes, given the difficulties to appreciate subtle neurological signs, such as mild weakness or sensory deficits. Furthermore, patient's difficulties to access the teleconsultation and the necessity of a caregiver for TM evaluation for some patients should be considered. Finally, clinical data obtained by TM could not be easily compared to those recorded during ambulatory visits. In this scenario, the use of clinical scales suitable to be administered via TM could help in NMD patient follow-up. An ideal scale for TM should be concise, easy to be administered, reproducible, comprehensive enough in capturing the wide range of different clinical manifestations and informative in remote evaluation of functions without direct clinical examination.

Facing the problem of TM consulting for NMD during COVID-19 outbreak, we suggest, as suitable for that, the following scales: ALSFR-R for motor neuron diseases [7], MG-ADL for neuromuscular junction diseases [8], MyoFRS for myopathies and Nerve-FRS (N-FRS) for neuropathies. ALSFR-R and MG-ADL are disease-specific scales validated for amyotrophic lateral sclerosis and myasthenia gravis, respectively; they appear the most reliable scales for a remote evaluation in these two disorders, as they satisfy the aforementioned prerogatives required in a TM context and in addition, they allow to compare the score obtained by TM with those previously obtained. Their use could be also extended to other motor neuron diseases and myasthenic syndromes, for which, other disease-specific scales could have some limitations by TM. Validated scales for myopathies and neuropathies are quite disease-specific and difficult to use in remote consultation due to a number of predominantly examiner-dependent items. Accordingly, we conceived the Myo-FRS and the N-FRS, two functional scales, 


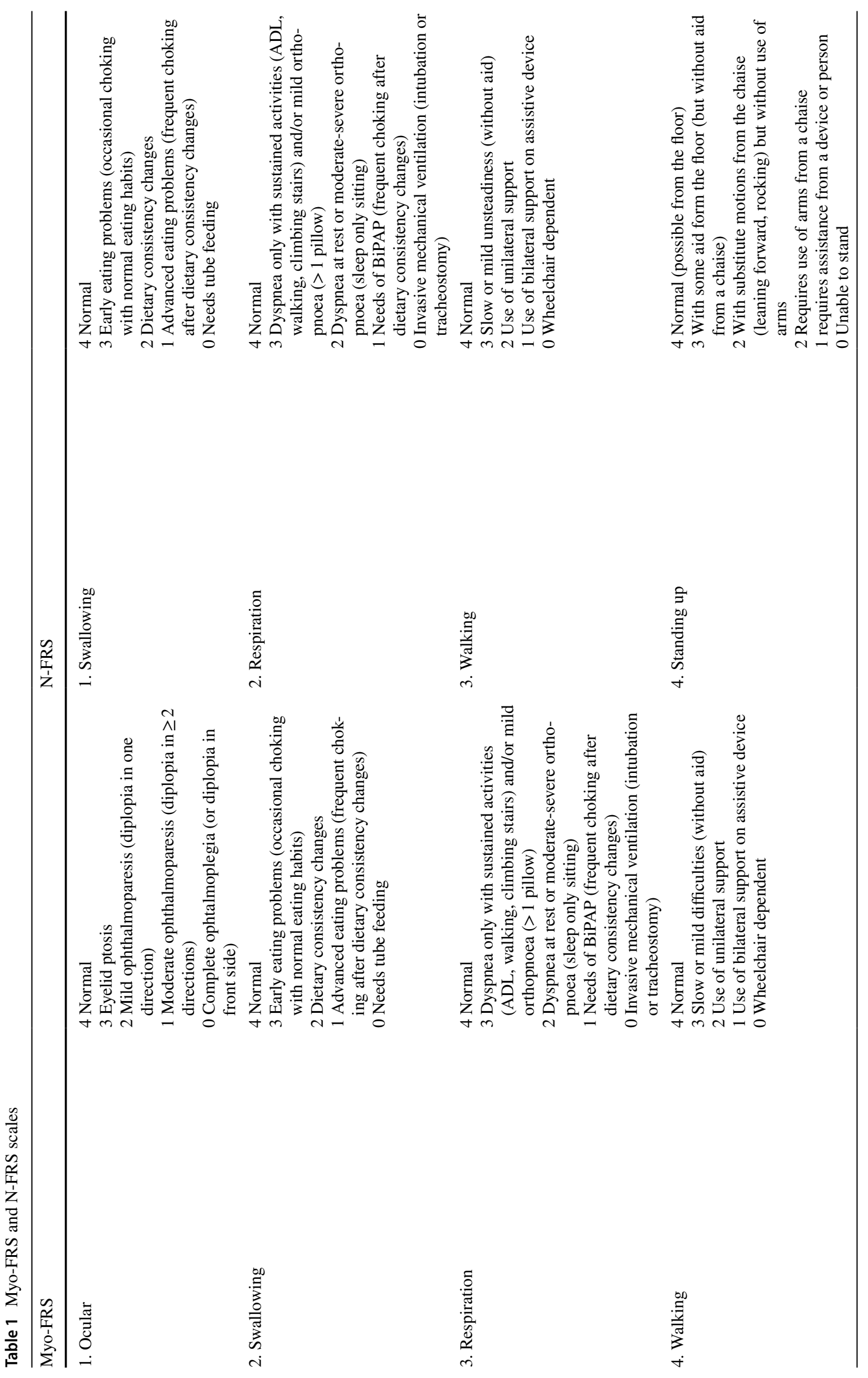




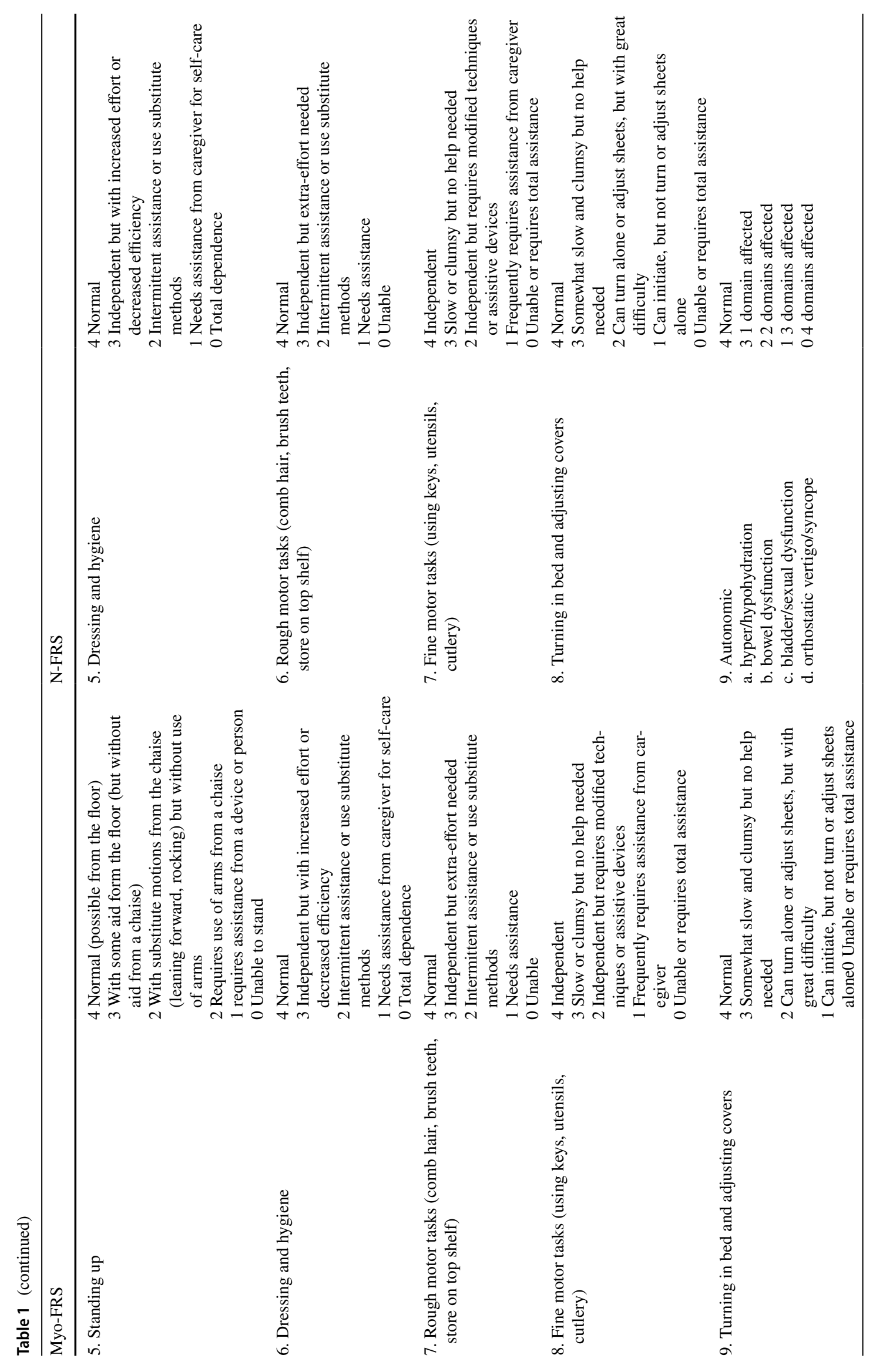




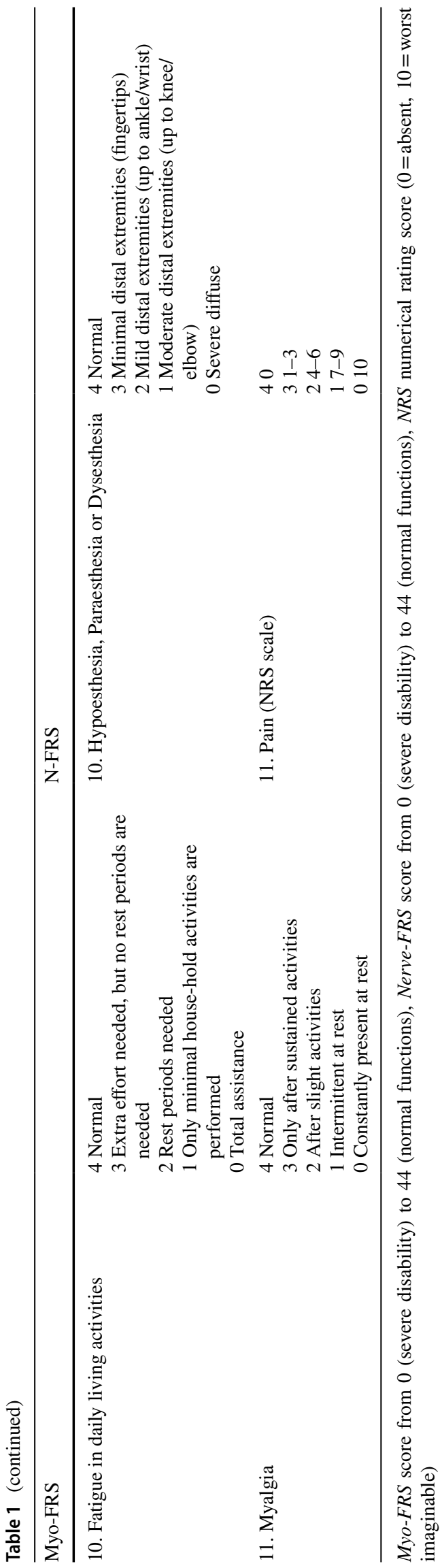

which, rather than explore too much disease-specific tasks, are aimed to capture, basically through a functional questionnaire, the overall characteristics of neuromuscular performance, both in myopathies and in neuropathies (Table 1). MYO-FRS and N-FRS have never been used before, and do not allow a comparison with formerly collected clinical data. However, they provide a practical and useful tool to assign a functional score, which reflects the overall neuromuscular impairment along the disease course. Certainly, time has to come for their validation, but in this historical moment, implementation of routine tests is urgently needed for a homogeneous and effective approach to NMD through TM.

\section{Compliance with ethical standards}

Conflicts of interest All authors report no relevant disclosures and conflict of interest for this study.

Ethical standard This study has been performed in accordance with the ethical stadards requirements

\section{References}

1. Lurie N, Carr BG (2018) The role of telehealth in the medical response to disasters. JAMA Internal Med 178:745-746. https:// doi.org/10.1001/jamainternmed.2018.1314

2. Ateev M, Kristin R, Brockmeyer DM et al (2020) Rapidly converting to "virtual practices": outpatient care in the era of Covid-19. Catalyst non-issue content 1. https://doi.org/10.1056/CAT.20.0091

3. Greenhalgh T, Koh GCH, Car J (2020) Covid-19: a remote assessment in primary care. BMJ 368:m1182. https://doi.org/10.1136/ bmj.m1182

4. Hollander JE, Carr BG (2020) Virtually perfect? telemedicine for Covid-19. N Engl J Med. https://doi.org/10.1056/NEJMp2003539

5. Hatcher-Martin JM, Adams JL, Anderson ER et al (2020) Telemedicine in neurology: telemedicine work group of the American Academy of Neurology update. Neurology 94:30-38. https://doi. org/10.1212/WNL.0000000000008708

6. Guidon AC, Amato AA (2020) COVID-19 and neuromuscular disorders. Neurology. https://doi.org/10.1212/WNL.0000000000 009566

7. Cedarbaum JM, Stambler N, Malta E et al (1999) The ALSFRS-R: a revised ALS functional rating scale that incorporates assessments of respiratory function. BDNF ALS study group (phase III). J Neurol Sci 169:13-21. https://doi.org/10.1016/s0022 $-510 x(99) 00210-5$

8. Wolfe GI, Herbelin L, Nations SP et al (1999) Myasthenia gravis activities of daily living profile. Neurology 52:1487-1489. https ://doi.org/10.1212/wnl.52.7.1487 